Methodology article

Open Access

\title{
Improving detection of differentially expressed gene sets by applying cluster enrichment analysis to Gene Ontology Tao $\mathrm{Xu}^{1,2}$, JianLei Gu${ }^{3}$, Yan Zhou*3,2 and LinFang Du*1
}

\author{
Address: ${ }^{1}$ College of Life Sciences, Sichuan University, Chengdu 610064, PR China, ${ }^{2}$ Shanghai-MOST Key Laboratory of Health and Disease \\ Genomics, Chinese National Human Genome Center at Shanghai, Shanghai 201203, PR China and ${ }^{3}$ Department of Microbiology, School of Life \\ Sciences, Fudan University, Shanghai 200433, PR China \\ Email: Tao Xu - xutao@chgc.sh.cn; JianLei Gu - jianleigu@gmail.com; Yan Zhou* - zhouy@chgc.sh.cn; LinFang Du* - dulinfang@yahoo.com \\ * Corresponding authors
}

Published: 5 August 2009

BMC Bioinformatics 2009, 10:240 doi:10.1186/1471-2105-10-240
Received: 14 January 2009

Accepted: 5 August 2009

This article is available from: http://www.biomedcentral.com/I47I-2/05/10/240

(C) 2009 Xu et al; licensee BioMed Central Ltd.

This is an Open Access article distributed under the terms of the Creative Commons Attribution License (http://creativecommons.org/licenses/by/2.0), which permits unrestricted use, distribution, and reproduction in any medium, provided the original work is properly cited.

\begin{abstract}
Background: Gene set analysis based on Gene Ontology (GO) can be a promising method for the analysis of differential expression patterns. However, current studies that focus on individual GO terms have limited analytical power, because the complex structure of $G O$ introduces strong dependencies among the terms, and some genes that are annotated to a GO term cannot be found by statistically significant enrichment.

Results: We proposed a method for enriching clustered $G O$ terms based on semantic similarity, namely cluster enrichment analysis based on $\mathrm{GO}(\mathrm{CeaGO})$, to extend the individual term analysis method. Using an Affymetrix HGU95aV2 chip dataset with simulated gene sets, we illustrated that CeaGO was sensitive enough to detect moderate expression changes. When compared to parentbased individual term analysis methods, the results showed that CeaGO may provide more accurate differentiation of gene expression results. When used with two acute leukemia (ALL and ALL/AML) microarray expression datasets, CeaGO correctly identified specifically enriched GO groups that were overlooked by other individual test methods.

Conclusion: By applying CeaGO to both simulated and real microarray data, we showed that this approach could enhance the interpretation of microarray experiments. CeaGO is currently available at http://chgc.sh.cn/en/software/CeaGO/.
\end{abstract}

\section{Background}

Identifying differentially expressed genes (DEGs) from microarray experiments enables researchers to elucidate related biological processes. In addition to studies focused on individual genes such as SAM[1], statistical techniques have been successfully employed to determine whether predefined groups, for example those in Gene Ontology (GO) [2], or in a metabolic pathway, are differentially expressed. There are two main statistical testing approaches: individual gene analysis (IGA) $[3,4]$ and Gene Set Analysis (GSA) [5]. IGA is performed in two steps: first, genes of interest are selected using a cutoff threshold, and the enriched biological categories are gained by statistically testing these genes against the background: typically all genes in the category (e.g., Fisher's exact test). The major limitation of IGA is that the result is significantly affected by an arbitrarily chosen cutoff in the first step. Hence, the GSA approach was developed to address this issue. GSA methods calculate a score based on all the genes within the gene set. Since it is free of the problems of threshold-based methods, GSA should be more sensitive than IGA, thus identifying gene sets with 
'subtle but coordinated' expression changes that cannot be detected by IGA. In previous studies, gene sets were formatted and pre-defined into groups such as independent GO terms and reference KEGG [6] pathways. Little attention was paid to subtle but coordinated expression changes across gene sets or within gene sets. In other words, the structure of gene sets was usually ignored.

Some authors have proposed taking into account the structure of gene sets when testing for gene set enrichment. Draghici et al. [7] developed an impact analysis of a signaling pathway that incorporates some crucial factors, such as each gene's position in the given pathway and their interactions. A few studies reported that through an intersect operation on gene sets between different categories, the gene sets could more precisely characterize the biological themes, and more closely represent the true differential expression functions of the data. The SEGS [8] and ADGO [9] methods attempted to improve gene set enrichment analysis by intersecting two different GO categories. Enrichment of GO terms with $p$-values calculated by the IGA method based on its neighbourhood using GO graph topology was also implemented in TopGO [10]. A few attempts $[10,11]$ have been proposed to address the redundant enrichment problem caused by overlapping annotations according to the fact that GO terms within a Directed Acyclic Graph (DAG) represent an inheritance relationship. We abstracted the simplest common opinion of these methods, called the parent-based approach which was implemented in the CeaGO package, was to join the genes of children to the parent GO term stepwise, and then tested all the GO terms in the graph topology. Elim and weight approaches presented in the study of Alexa et al. [10] and parent-child in Grossmann et al. [11] are complicated but useful for minimizing false positives and can enrich GO terms more accurately. Otherwise, if an insufficient number of genes are annotated to one GO term, methods may not be sensitive enough to uncover subtle expression changes, similar to the drawbacks of significance analysis of individual genes [12]. Thus, our goal was to identify some novel expression changes by grouping GO terms. Similarity between pairs of GO terms provided an opportunity to enlarge the GO groups to better interpret the gene expression data.

In this article, we present an effective method, cluster enrichment analysis based on GO (CeaGO), to extend enrichment analysis of individual GO terms and discover significantly clustered GO classes from expression data. A sufficiently rigorous standard could not be found to evaluate the algorithm; instead, a simulation study was performed to assess the statistical properties of this method. Finally, we applied this method to the gene expression profiles of ALL [13] and ALL/AML datasets [14]. The results from both simulated and real data showed that
CeaGO is sensitive enough to identify significant expression changes overlooked by individual term tests.

\section{Methods}

Figure 1 illustrates the overall procedure of CeaGO.

\section{Semantic similarity calculation}

Using groups of genes from microarray experiments, we assigned each gene to the GO terms. For the induced GO graph, we measured the relationship of two GO terms. A variety of algorithms can compute semantic similarity between terms [15-18]. We used the simple but effective measure developed by Resnik [17]. GO allows two terms to share parents. Given all parents (denoted $S\left(t_{1}, t_{2}\right)$ ) of two query terms $t_{1}$ and $t_{2}$, Resnik defined semantic simi-

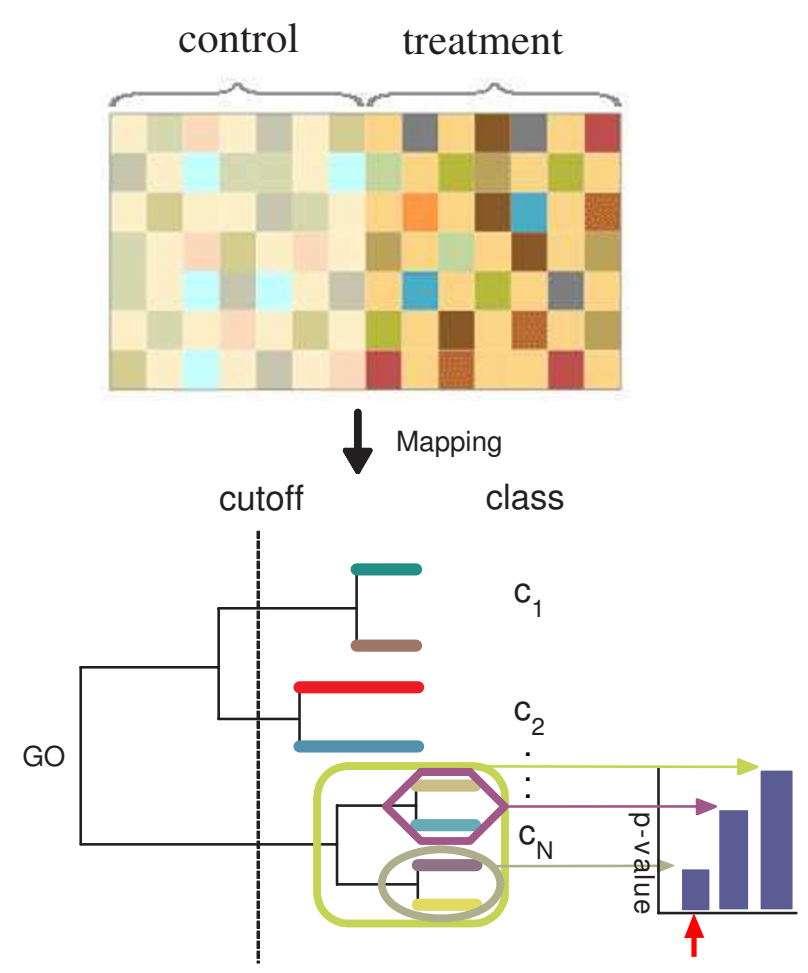

Figure I

An overview of the CeaGO method. The upper panel shows the expression of genes in two classes, for example, gene expression in control versus treatment. After genes are annotated to the GO DAG, a dendrogram is generated using a hierarchical clustering method based on semantic similarity. For such a dendrogram, many classes $\left(C_{1}, \ldots, C_{N}\right)$ are obtained when the tree meets a user-defined cutoff (lower left panel). The $p$-values are computed according to the gene set enrichment analysis for every subset of each class. The lower right panel shows those $p$-values in a histogram against each subset of the class. The minimum (red arrow) is found and is used to determine the subset (grey green ellipse) of the given class. Such subsets would be assigned as "most likely to be differentially expressed" in the microarray data. 
larity between two GO terms as the information content of the term with minimum probability (denoted $p(t)$ ) among the common ancestors.

$$
\operatorname{sim}\left(t_{1}, t_{2}\right)=-\ln \min _{t \in S\left(t_{1}, t_{2}\right)} p(t)
$$

The GO similarity score can be transformed into a GO distance $d\left(t_{1}, t_{2}\right)$ :

$$
d\left(t_{1}, t_{2}\right)=-\ln \min _{c \in S(t)} p(t)-\operatorname{sim}\left(t_{1}, t_{2}\right)
$$

Note that $S(t)$ in this definition is the set of all terms in one ontology.

\section{Hierarchical clustering}

Hierarchical clustering is a multiple step, agglomerative method that sequentially merges samples based on the pair-wise similarity of a given measurement, forming common partitions until all samples are contained in a single group. At each particular stage, the method joins together the two previous clusters that are closest together (most similar). A dendrogram is built by using the semantic similarity metric described above. $N$ classes are obtained when the tree meets a user-defined similarity distance threshold (in this study taken as $d<2$ ). The model of each class can be described as a vector $\vec{G}=\left(G_{1}\right.$, $\left.G_{2}, \ldots, G_{n}\right)$, where $G_{i}$ represents the genes annotated to GO terms obtained at the $i^{\text {th }}$ step in the clustering process. When such a class is formed, various statistical tests can be used to determine whether the genes within the $G_{i}$ showed coordinated expression.

\section{Gene set enrichment analysis}

To detect possibly moderate but coordinated expression changes within a gene set, we employed a simple but robust $Z$-statistic method named PAGE [19] from several available GSA algorithms [12,20-23]. The Z-score is calculated according to the following equation:

$$
z=(x-\mu) * \sqrt{n} / \sigma
$$

Where is the mean of total fold change [24] values and is the standard deviation of total fold change values of a given microarray data set, $x$ is the mean of the fold change values and $n$ is the total number of genes in the gene set. Fold changes are calculated for all genes between two experimental groups (e.g. control versus treatment). $P$-values inferred from $Z$-scores against standard normal distribution are calculated. According to PAGE, 10 samples should be sufficiently close to normal distribution and provide a fairly good statistical test. Therefore, gene sets larger than 10 were used to test the differential gene expression changes.

Next, a vector of significant $p$-values, which was described as $\vec{P}=\left(p_{1}, p_{2}, \ldots, p_{n}\right)$, associated with $\vec{G}$ was computed for each GO class. If the $p$-values were less than a pre-defined value (e.g. < 0.05), those gene sets were considered as significantly differentially expressed. When such gene sets were found, the gene sets were reduced by retaining the GO subsets with the smallest $p$-values. At this point, the biologically meaningful sets were identified by the tally of GO subsets selected during this step.

The algorithms were implemented in the R programming language http://www.r-project.org. The results were obtained using R version 2.7.1 and the libraries provided by the Bioconductor project http://www.bioconduc tor.org, version 2.2.

\section{Results}

\section{Validation of CeaGO on simulated data}

The evaluation of enrichment measurement methods is a challenging task, because biologically meaningful gene sets usually are not known for real datasets. In this study, we introduced an evaluation framework similar to one described previously [10] to address this issue. To imitate real data as closely possible, an artificial data set derived from a HGU95aV2 chip with all 10,503 probes representing genes annotated by terms from the GO biological process subontology was used, and the resulting graph with 4,913 nodes was used as the underlying dataset. Here, we presumed that the expression values of the control and the treatment groups obeyed a standard normal distribution $N(0,1)$. After clustering the GO terms, 188 classes, annotated with more than 10 genes each, were obtained by cutting the dendrogram $(d<2)$. We selected 25 "truly enriched" GO classes at random from the 188 classes. One set was chosen randomly from the subsets in each GO class and denoted as the "truly" differentially expressed gene set. Given those gene sets, $N(0,1)$ distributed genes in the treatment groups were replaced by $N(, 1)$ distributed genes, with the genes in the control groups remaining as $N(0,1)$. The test of dynamic change of was not performed in this study because $Z$-statistic methods are not sensitive enough to detect changes of sample standard deviation (see Eq. (3)).

Instead of focusing on a list of differentially expressed groups arbitrarily determined by a pre-defined threshold, we were interested in the groups at the top of the list. Therefore, after enrichment of the GO sets, the enriched groups were sorted in ascending order of their $p$-values. 
Assuming $S_{t}$ denotes the set of "truly enriched" GO sets, and $E_{t}$ denotes the set of top-scoring GO groups enriched by CeaGO of the same size as $S_{t}$ an "Exact match" score was used to evaluate the performance of CeaGO:

$$
\text { Score }=\operatorname{count}\left(E_{t} \cap S_{t}\right)
$$

The score is the number of pre-selected GO sets found among the top $k$ enriched sets. It lies in the interval $[0, k]$, where $k$ is the perfect prediction by this method.

Another score was also implemented for evaluating the detecting power of the "Possible match" members. Such members represent the fraction of perfect matchings of the pre-selected "truly enriched" GO sets, and the "in but wrong" members that failed, but were still detected as the same class of the "truly enriched" GO sets.

The effect of changing of genes in the treatment groups over the 100 permutations is shown in Figure 2. We observed that detection of both "Possible match" and "Exact match" gene sets exhibited a trend in which larger had the higher scores. This increasing trend tended to be unclear when the value was above 0.4. The "Possible match" scores indicated that the PAGE algorithm was sensitive enough to detect differentially expressed groups. The results obtained by this approach showed that most

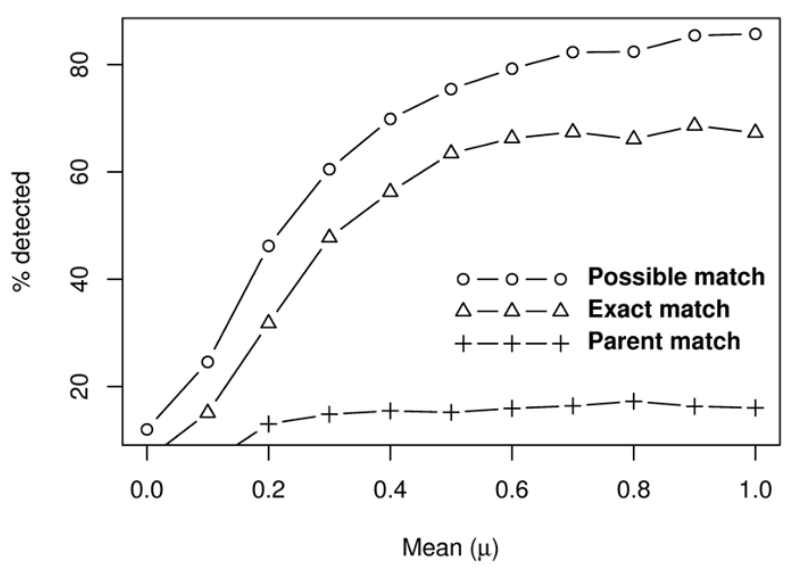

\section{Figure 2}

Effect of changing on simulated data. This figure illustrates how results change when the is changed. "Exact match" represents the percentage of GO groups enriched by the CeaGO exact match to the pre-selected "truly enriched" gene sets. The "Possible match" groups are those that occur in the pre-selected GO classes. The "Parent match" indicates the percentage of top nodes enriched by the parent-based enrichment method found among the most recent parent nodes of the pre-selected "truly enriched" GO sets. of the "Exact match" gene sets were detectable (about $70 \%)$ at a high $(>0.4)$. About $20 \%$ of the "in but wrong" gene sets failed to match the correct GO sets precisely. However, the observed classes might still be helpful in explaining the differentially expressed groups. The "Parent match" scores were calculated to describe the efficacy of CeaGO against the parent-based enrichment methods. Only about $15 \%$ of GO terms appeared in the pool of most recent parent nodes in 25 pre-selected classes (Figure 2 ). We have performed the same analysis for one of the parent-based methods Elim [10]. The results we obtained were similar to those described above. For example, the detecting power of "Parent match" was about $40 \%$ above a certain value of (about 0.6) [see Additional file 1]. These results indicated that the proposed procedure was able to enrich for the correct, differentially expressed gene sets.

\section{Application of CeaGO to ALL data sets}

CeaGO was first applied to the well-known expression dataset Acute Lymphocytic Leukemia (ALL) developed by Chiaretti et al. [13]. These data were collected to characterize the relationship between gene expression signatures in ALL-associated cells and genotypic abnormalities in adult patients and the dataset is available from Bioconductor [25]. One use of the dataset has been to examine B-cell lines with this disease and find differential gene expression between the $\mathrm{BCR} / \mathrm{ABL}$ samples that have rearrangements in the BCR/ABL genes, and NEG samples, which have no evidence of major molecular rearrangements. There are 37 samples for the BCR/ABL group and 42 for the NEG group, each of which has been hybridized to an Affymetrix HGU95Av2 chip containing 12,625 gene-associated probes. We began by normalizing the dataset using the variance stabilizing method VSN [26]. Subsequently, 10,503 genes were successful mapped to GO terms from the BP ontology yielding a list of genes with a GO graph of 3,066 terms.

CeaGO identified five GO clusters with significant $p$-values $(<0.05)$. The raw $p$-values were adjusted using the false discovery rate (FDR) method from Benjamini and Yekutieli [27]. Table 1 presents some novel significant groups that were discarded by individual GO term analysis. For example, genes categorized by $S$ phase of mitotic cell cycle: 0000084\&\& regulation of transcription during S-phase of mitotic cell cycle: 0000115 was significantly differentially expressed in the list scored by CeaGO $(p$-value $=2.9 e-5)$. According to an earlier report [28], the $\mathrm{BCR} / \mathrm{ABL}$ protein translocates to the nucleus and disrupts an ATR-dependent intra-S phase checkpoint. We observed that genes categorized by activation of JUN kinase activity: 0007257\&\& positive regulation of JUN kinase activity: 0043507 were significantly induced, but neither exhibited significant differential expression using individual GO term analysis. This is consistent with the report that $\mathrm{BCR} / \mathrm{ABL}$ leukemia onco- 
Table I: Top significant GO groups identified between BCR/ABL and NEG phenotypes for the ALL dataset.

\begin{tabular}{|c|c|c|c|c|}
\hline No & GO ID & Term & Rank $^{\mathrm{a}}$ & $p$-value \\
\hline \multirow[t]{3}{*}{1} & GO:0043122 & regulation of I-kappaB kinase/NF-kappaB ... & 3 & $3.7 e-06$ \\
\hline & GO:0043I23 & positive regulation of I-kappaB kinase/N... & - & \\
\hline & GO:0043124 & negative regulation of I-kappaB kinase/N... & - & \\
\hline \multirow[t]{2}{*}{2} & GO:0000084 & S phase of mitotic cell cycle & - & $2.9 e-05$ \\
\hline & GO:0000II5 & S-phase-specific transcription in mitoti... & - & \\
\hline \multirow[t]{5}{*}{3} & GO:00327I5 & negative regulation of interleukin-6 pro... & - & 0.012 \\
\hline & GO:0032755 & positive regulation of interleukin- 6 pro... & - & \\
\hline & GO:0042226 & interleukin- 6 biosynthetic process & - & \\
\hline & GO:0045408 & regulation of interleukin- 6 biosynthetic... & - & \\
\hline & GO:00454I0 & positive regulation of interleukin- 6 bio... & - & \\
\hline \multirow[t]{3}{*}{4} & GO:0032088 & inhibition of NF-kappaB transcription fa... & - & 0.027 \\
\hline & GO:0043392 & negative regulation of DNA binding & - & \\
\hline & GO:0043433 & negative regulation of transcription fac... & - & \\
\hline \multirow[t]{2}{*}{5} & GO:0007257 & activation of JNK activity & - & 0.045 \\
\hline & GO:0043507 & positive regulation of JNK activity & - & \\
\hline
\end{tabular}

a Refers to the rank on the list of top GO terms enriched by individual GO term analysis for FDR controlled at $5 \%$. The dash (-) indicates that this $\mathrm{GO}$ term was not found on the list generated by individual GO term analysis.

gene activates Jun kinase and requires Jun for transformation [29]. The accuracy of the enriched GO cluster method for interleukin- 6 was also supported by previous research [30].

The disadvantage with parent-based enrichment studies is that they use only the most recent parent node to calculate the significance of gene sets. Therefore, we grouped the GO terms into a cluster, which was a feasible solution for improving the sensitivity of our programme. For example, Figure 3 shows a subgraph induced by $S$ phase of mitotic cell cycle: 0000084\&\& regulation of transcription during S-phase of mitotic cell cycle: 0000115 that exhibited significant differential expression by CeaGO. In this subgraph, the Benjamini and Yekutieli adjusted $p$-value of PAGE analysis of $S$ phase of mitotic cell cycle: 0000084 using all genes with GO terms was only 0.025 ( $p$-value $=0.0068$ in elim), which was much less significant than the result of CeaGO analysis $(p$-value $=2.9 \mathrm{e}-5)$. Some groups returned similar results to the parent-based method, however. For example, activation of JUN kinase activity: 0007257\&\& positive regulation of JUN kinase activity: 0043507 had an adjusted pvalue of 0.045 , while the parent node positive regulation of JUN kinase activity: 0043507 had an adjusted $p$-value of 0.038 . In these cases, cluster members identified by CeaGO were almost the same as the offspring of the GO term enriched by parent-based analysis, which lead to identical significant score. These results indicate that the CeaGO method is more sensitive at detecting certain novel expression changes than parent-based enrichment methods, while some coordinated changes were preserved.

\section{Application of CeaGO to the ALLIAML dataset}

The purpose of the present study was to examine the applicability of the CeaGO algorithm. In addition, we tested the algorithm with a published dataset called golubEsets [14], which is also available from Bioconductor. It consists of 7,129 genes from 47 samples of acute lymphoblastic leukemia (ALL), and 25 samples of acute myeloblastic leukemia (AML). Normalization on these samples was also carried out using VSN. This pre-processing resulted in 6,372 genes annotated to GO terms from BP ontology. The induced GO graph contains 2,766 GO terms.

The enriched GO clusters scored by CeaGO are summarized in Table 2. Four clusters had differential gene expression levels in ALL versus AML at the significance level of 0.05 . $P$-values were adjusted with the FDR procedure (Benjamini and Yekutieli [27]). CeaGO analysis reported "chemokine" as highly significant from a statistical perspective $(p$-value $=9 \mathrm{e}-09$ for cluster NO. 1 , and 3.4e-06 for cluster NO. 2). This is consistent with previous research showing that chemokines affect the proliferation of AML cells and that primary AML cells constitutively release chemokine [31]. Moreover, differences in chemokine responsiveness, as well as chemokine release, are reported to contribute to patient heterogeneity in AML [32]. The second cluster as ranked by the CeaGO analysis concerned "tumor necrosis factor" (FDR corrected $p$-value $=0.0014$ ). The importance of this cluster is well-supported. For example, tumor necrosis factor alpha (TNF $\alpha)$ can increase the proliferation of AML cells [33]. 


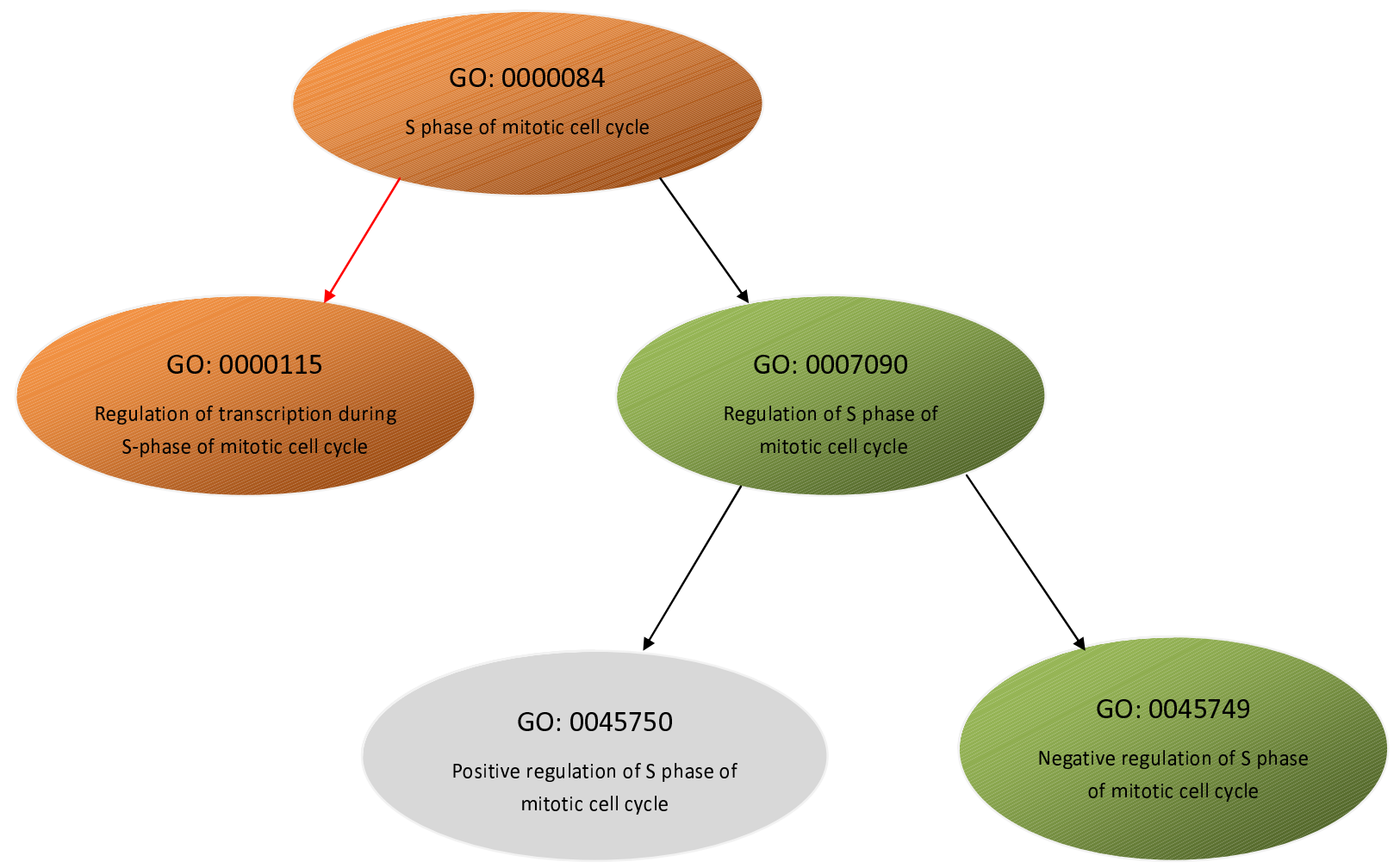

Figure 3

Subgraph for $\mathbf{G O}$ term $S$ phase of mitotic cell cycle: $\mathbf{0 0 0 0 0 8 4}$. Orange circles represent significant clusters enriched by CeaGO, green ellipses stand for the annotated GO terms in ALL data but not enriched by CeaGO, and grey ellipse represents ALL genes not mapped to GO terms. Black arrows indicate is-a relationships and red arrow indicates part-of relationship.

Table 2: Top significant GO groups identified between AML and ALL for the ALL/AML dataset.

\begin{tabular}{|c|c|c|c|c|}
\hline No & GO ID & Term & Rank $^{\mathrm{a}}$ & $p$-value \\
\hline \multirow[t]{4}{*}{ I } & GO:0042033 & chemokine biosynthetic process & - & $9 e-09$ \\
\hline & GO:0045079 & negative regulation of chemokine biosynt... & - & \\
\hline & GO:0045080 & positive regulation of chemokine biosynt... & - & \\
\hline & GO:0050754 & positive regulation of fractalkine biosy... & - & \\
\hline \multirow[t]{2}{*}{2} & GO:0050927 & positive regulation of positive chemotax... & - & $3.4 \mathrm{e}-06$ \\
\hline & GO:0050930 & induction of positive chemotaxis & - & \\
\hline \multirow[t]{4}{*}{3} & GO:0032720 & negative regulation of tumor necrosis fa... & - & 0.0014 \\
\hline & GO:0032760 & positive regulation of tumor necrosis fa... & - & \\
\hline & GO:0042535 & positive regulation of tumor necrosis fa... & - & \\
\hline & GO:0042536 & negative regulation of tumor necrosis fa... & - & \\
\hline \multirow[t]{3}{*}{4} & GO:0045807 & positive regulation of endocytosis & - & 0.0058 \\
\hline & GO:0048260 & positive regulation of receptor-mediated... & - & \\
\hline & GO:0050766 & positive regulation of phagocytosis & - & \\
\hline
\end{tabular}

a Refers to the rank on the list of the top GO terms enriched by individual GO term analysis for FDR controlled at $5 \%$. The dash (-) indicates that the GO term was not found on the list generated by individual GO term analysis. 


\section{Discussion}

Traditional strategies for gene expression analysis have focused on identifying individual genes or pre-defined groups such as those in KEGG pathways and Gene Ontology, which exhibit differences between two states of interest. In this article, we extended expression analysis from prior defined gene sets to a gene set analysis framework that makes use of the structure of GO.

GO has a hierarchical structure that forms a DAG. CeaGO uses the clustering method to combine similar GO terms, rather than using the parent terms, and successfully detected several novel, meaningful categories. One objective of these methods is to enlarge the gene sets to enable identification of new, differentially expressed groups. Methods derived from a parent term with all the genes of its children may introduce unnecessary noise, and fail to detect significant changes. The superior performance of CeaGO than parent-based method using PAGE gene set enrichment algorithm is supported by the results presented here on simulated and ALL data. Similar scenarios were observed with the ALL/AML dataset. However, the number of GO groups enriched by the CeaGO method is related to the cutoff of semantic distances. If the threshold was set to the maximum distance of two GO terms of the ontology, CeaGO gave just one cluster, which is too biologically general. If the threshold was set to 0, all GO terms were independent, which is the same as the individual GO term analysis. Fortunately, we found that CeaGO is not sensitive to this parameter, and an arbitrary $20 \%$ of the maximum semantic distance was chosen to balance biological meaning and cluster numbers.

The application of CeaGO relies on the accuracy of the gene set test methods. Fortunately, the proposed method operates within a well-defined statistical framework, so that other statistical tests for assessing the significance of GO sets can be used with CeaGO, for example GSEA [12] or GlobalANCOVA [21]. The Z-statistic algorithm was employed in this article because of its fast computation advantage over the permutation-based methods. For example, PAGE reduces computation time at least 5,000fold when performing 5,000 dataset permutations to get a background distribution. However, it suffers from limitations on the normal distribution hypothesis and the minimal size of gene sets. As the accuracy of statistical test methods increases, the performance of CeaGO should improve, as applied to experimental datasets.

In many, if not all cases, analysis with individual GO terms may not be sufficient to reveal changes in specific expression patterns. For example, not all the genes annotated to a "Biological Process" term may exhibit as differentially expressed, but only those with a particular localization such as "membrane" might alter their expression. Through intersection of gene sets between different categories, gene sets can be separated more specifically, facilitating a much more detailed analysis of expression patterns $[8,9]$. In future studies, we will introduce a comprehensive algorithm to investigate genes within a single GO term or a clustered GO class, with the goal of uncovering the truly differential expression functions in expression data.

\section{Conclusion}

Gene set analysis based on GO is a popular and useful approach to extract biological information from expression data. However, this is limited from showing its full analytical power when only pre-defined GO terms are used, because an insufficient number of genes annotated to one GO term may not be sensitive enough to detect subtle expression changes. Therefore, we developed a novel method to extend the traditional individual GO term analysis. This method dynamically enriches clustered GO terms to identify groups that are significantly differentially expressed in microarray data. Compared to individual GO term analysis (including parent-based enrichment methods), the results obtained from both simulated and real microarray data sets showed that the proposed approach is very promising. Furthermore, the CeaGO model can easily be extended to other gene set analysis methods.

\section{Availability and requirements}

Project name: CeaGO

Project homepage: http://chgc.sh.cn/en/software/CeaGO/

Operating system(s): Platform Independent

Programming languages: $\mathrm{R}$ and $\mathrm{C}$

\section{Authors' contributions}

TX, YZ and LD jointly designed the CeaGO method. TX implemented the software. TX and JG performed the bioinformatics analyses. TX and YZ drafted the manuscript. All authors read and approved the final manuscript.

\section{Additional material}

\section{Additional file 1}

Effect of changing $\mu$ on simulated data. This figure illustrates how results change when the $\mu$ is changed. "Exact match" represents the percentage of GO groups enriched by the CeaGO exact match to the preselected "truly enriched" gene sets. The "Possible match" groups are those that occur in the pre-selected GO classes. The "Parent match" indicates the percentage of top nodes enriched by the elim enrichment method found among the most recent parent nodes of the pre-selected "truly enriched" GO sets.

Click here for file

[http://www.biomedcentral.com/content/supplementary/14712105-10-240-S1.pdf] 


\section{Acknowledgements}

The work was supported by the National Natural Sciences Foundation of China (No.30870 I I8), the Doctoral Foundation of Ministry of Education of China (No.200706 10080), Program for New Century Excellent Talents in University (NCET-04-086I), Sichuan University Research Grant 985 and China National High-tech 863 Program (2006AA02Z335).

\section{References}

I. Tusher VG, Tibshirani R, Chu G: Significance analysis of microarrays applied to the ionizing radiation response. Proceedings of the National Academy of Sciences of the United States of America 200I, 98(9):5I|6-5|2|.

2. Ashburner M, Ball CA, Blake JA, Botstein D, Butler H, Cherry JM, Davis AP, Dolinski K, Dwight SS, Eppig JT, et al.: Gene ontology: tool for the unification of biology. The Gene Ontology Consortium. Nature genetics 2000, 25(I):25-29.

3. Khatri P, Draghici S: Ontological analysis of gene expression data: current tools, limitations, and open problems. Bioinformatics (Oxford, England) 2005, 2 I ( I 8):3587-3595

4. Rivals I, Personnaz L, Taing L, Potier MC: Enrichment or depletion of a GO category within a class of genes: which test? Bioinformatics (Oxford, England) 2007, 23(4):40I-407.

5. Nam D, Kim SY: Gene-set approach for expression pattern analysis. Briefings in bioinformatics 2008, 9(3): I89-197.

6. Ogata H, Goto S, Sato K, Fujibuchi W, Bono H, Kanehisa M: KEGG: Kyoto Encyclopedia of Genes and Genomes. Nucleic acids research 1999, 27(I):29-34

7. Draghici S, Khatri P, Tarca AL, Amin K, Done A, Voichita C, Georgescu C, Romero R: A systems biology approach for pathway level analysis. Genome research 2007, I 7( I 0): I537-I545

8. Trajkovski I, Lavrac N, Tolar J: SEGS: search for enriched gene sets in microarray data. Journal of biomedical informatics 2008 4 I(4):588-60I.

9. Nam D, Kim SB, Kim SK, Yang S, Kim SY, Chu IS: ADGO: analysis of differentially expressed gene sets using composite GO annotation. Bioinformatics (Oxford, England) 2006 , 22( I 8):2249-2253.

10. Alexa A, Rahnenfuhrer J, Lengauer T: Improved scoring of functional groups from gene expression data by decorrelating GO graph structure. Bioinformatics (Oxford, England) 2006 22(13): I600-1607.

II. Grossmann S, Bauer S, Robinson PN, Vingron M: Improved detection of overrepresentation of Gene-Ontology annotations with parent child analysis. Bioinformatics (Oxford, England) 2007, 23(22):3024-303I.

12. Mootha VK, Lindgren CM, Eriksson KF, Subramanian A, Sihag S, Lehar J, Puigserver P, Carlsson E, Ridderstrale M, Laurila E, et al:: PGCI alpha-responsive genes involved in oxidative phosphorylation are coordinately downregulated in human diabetes. Nature genetics 2003, 34(3):267-273.

13. Chiaretti S, Li X, Gentleman R, Vitale A, Wang KS, Mandelli F, Foa R, Ritz J: Gene expression profiles of B-lineage adult acute lymphocytic leukemia reveal genetic patterns that identify lineage derivation and distinct mechanisms of transformation. Clin Cancer Res 2005, I I(20):7209-72I9.

14. Golub TR, Slonim DK, Tamayo P, Huard C, Gaasenbeek M, Mesirov JP, Coller H, Loh ML, Downing JR, Caligiuri MA, et al: Molecular classification of cancer: class discovery and class prediction by gene expression monitoring. Science (New York, NY) 1999. 286(5439):531-537.

I5. Jiang DW: Semantic Similarity Based on Corpus Statistics and Lexical Taxonomy. Proceeding of International Conference Research on Computational LinguisticsTaipei 1997:19-33.

16. Lin D: An Information-Theoretic Definition of Similarity. Proceedings of the Fifteenth International Conference on Machine Learning 1998.

17. Resnik P: Using Information Content to Evaluate Semantic Similarity in a Taxonomy. Proceedings of the 14th International Joint Conference on Artificial Intelligence 1995:448-453.

18. Wang JZ, Du Z, Payattakool R, Yu PS, Chen CF: A new method to measure the semantic similarity of GO terms. Bioinformatics (Oxford, England) 2007, 23(10): I274- I28I.

19. Kim SY, Volsky DJ: PAGE: parametric analysis of gene set enrichment. BMC bioinformatics 2005, 6: 144.
20. Tian L, Greenberg SA, Kong SW, Altschuler J, Kohane IS, Park PJ: Discovering statistically significant pathways in expression profiling studies. Proceedings of the National Academy of Sciences of the United States of America 2005, I 02(38): I 3544- I 3549.

21. Hummel M, Meister R, Mansmann U: GlobalANCOVA: exploration and assessment of gene group effects. Bioinformatics (Oxford, England) 2008, 24(I):78-85.

22. Dinu I, Potter JD, Mueller T, Liu Q, Adewale AJ, Jhangri GS, Einecke $G$, Famulski $K S$, Halloran $P$, Yasui $Y$ : Improving gene set analysis of microarray data by SAM-GS. BMC bioinformatics 2007, 8:242.

23. Breitling R, Amtmann A, Herzyk P: Iterative Group Analysis (iGA): a simple tool to enhance sensitivity and facilitate interpretation of microarray experiments. BMC bioinformatics 2004, 5:34

24. Choe S, Boutros M, Michelson A, Church G, Halfon M: Preferred analysis methods for Affymetrix GeneChips revealed by a wholly defined control dataset. Genome biology 2005, 6:RI6.

25. Gentleman RC, Carey VJ, Bates DM, Bolstad B, Dettling M, Dudoit S, Ellis B, Gautier L, Ge Y, Gentry J, et al:: Bioconductor: open software development for computational biology and bioinformatics. Genome biology 2004, 5( I 0):R80.

26. Huber $W$, von Heydebreck A, Sultmann $H$, Poustka A, Vingron $M$ : Variance stabilization applied to microarray data calibration and to the quantification of differential expression. Bioinformatics (Oxford, England) 2002, I 8(Suppl I):S96-104.

27. Benjamini $Y$, Yekutieli $D$ : The control of the false discovery rate in multiple testing under dependency. Ann Statist 200I, 29(4): I 165-1 I88.

28. Dierov J, Dierova R, Carroll M: BCR/ABL translocates to the nucleus and disrupts an ATR-dependent intra-S phase checkpoint. Cancer cell 2004, 5(3):275-285.

29. Raitano AB, Halpern JR, Hambuch TM, Sawyers CL: The Bcr-Abl leukemia oncogene activates Jun kinase and requires Jun for transformation. Proceedings of the National Academy of Sciences of the United States of America 1995, 92(25): I I 746- I I 750.

30. Cambier N, Zhang Y, Vairo G, Kosmopoulos K, Metcalf D, Nicola NA, Elefanty AG: Expression of BCR - ABL in MI myeloid leukemia cells induces differentiation without arresting proliferation. Oncogene 1999, I 8(2):343-352.

31. Olsnes AM, Motorin D, Ryningen A, Zaritskey AY, Bruserud O: T lymphocyte chemotactic chemokines in acute myelogenous leukemia (AML): local release by native human AML blasts and systemic levels of CXCL I 0 (IP- I 0), CCL5 (RANTES) and CCLI 7 (TARC). Cancer Immunol Immunother 2006, 55(7):830-840.

32. Bruserud O, Ryningen A, Olsnes AM, Stordrange L, Oyan AM, Kalland $\mathrm{KH}$, Gjertsen BT: Subclassification of patients with acute myelogenous leukemia based on chemokine responsiveness and constitutive chemokine release by their leukemic cells. Haematologica 2007, 92(3):332-34I.

33. Brailly $\mathrm{H}$, Pebusque MJ, Tabilio A, Mannoni P: TNF alpha acts in synergy with GM-CSF to induce proliferation of acute myeloid leukemia cells by up-regulating the GM-CSF receptor and GM-CSF gene expression. Leukemia I993, 7(I0):| 557-I 563.

Publish with Bio Med Central and every scientist can read your work free of charge

"BioMed Central will be the most significant development for disseminating the results of biomedical research in our lifetime. "

Sir Paul Nurse, Cancer Research UK

Your research papers will be:

- available free of charge to the entire biomedical community

- peer reviewed and published immediately upon acceptance

- cited in PubMed and archived on PubMed Central

- yours - you keep the copyright
BioMedcentral 